Running Header: SDT ANALYSES OF STATISTICAL CRITERIA

Insights into Criteria for Statistical Significance from Signal Detection Analysis

Jessica K. Witt

Colorado State University

Under peer review at Meta-Psychology. Editorial process can be accessed at: osf.io/69xmg.

Anyone can contribute with Open Peer Review. Contact the editor Daniel Lakens at

D.Lakens@tue.nl or contribute directly in the OSF project. 
What is best criterion for determining statistical significance? In psychology, the criterion has been $p<$ .05. This criterion has been criticized since its inception, and the criticisms have only heighted with recent failures to replicate studies published in top psychology journals. Several replacement criteria have been suggested including reducing the alpha level to .005 or switching to other types of criteria such as Bayes factors or effect sizes. Here, various criteria for statistical significance were evaluated using signal detection analysis on the outcomes of simulated data. With respect to the ability to discriminate between true effects and null effects, both p-values and Bayes factors resulted in fairly high discriminability, and performance was equivalent across both. Discriminability was better for effect size. With respect to bias, the specific thresholds that produced maximally-optimal utility depended on sample size, although this dependency was particularly notable for $p$-values and less so for Bayes factors. These simulations help illustrate some of the main themes regarding the interpretation of p-values and statistical significance. Importantly, the novel application of signal detection theory to the issue of statistical significance highlights the need to focus on both false alarms and misses, rather than false alarms alone. 
Insights into Criteria for Statistical Significance from Signal Detection Analysis

Scientists across many disciplines including psychology, biology, and economics use $p<.05$ as the criterion for statistical significance. This cut-off has recently been challenged due to numerous failures to replicate findings published in top journals (Begley \& Ellis, 2012; Camerer et al., 2016; Open Science Collaboration, 2015). Changes in the recommendations for statistical significance include using a stricter criterion for significance (e.g. p <.005; Benjamin et al., 2017), switching to Bayes factor (Dienes, 2011; Kruschke, 2011; Rouder, Speckman, Sun, Morey, \& Iverson, 2009), switching to effect sizes and confidence intervals (e.g. Cumming, 2014), and minimizing flexibility in decisions around data collection and analysis (e.g. Simmons, Nelson, \& Simonsohn, 2011). These recommendations were designed to increase replicability by decreasing the false alarm rates, which is the rate at which null effects are incorrectly labeled as significant. However, the recommendations have focused almost exclusively on replicability and false alarm rates with little-to-no discussion of discriminability. An ideal criterion for statistical significance is one that can best discriminate real effects from null effects. As shown below, data simulations revealed that none of the recommendations improved discrimination between real and null effects. Instead, these recommendations merely decrease false alarm rates while simultaneously increasing miss rates, which is the rate at which a real effect is judged as not significant. The best practice moving forward is for each discipline (or journal or scientist) to consider the relative costs of false alarms versus misses, and select a criterion for significance accordingly.

Failures to replicate prior research, the so-called replication crisis, has elicited many reactions. Among scientists, the reactions have been mixed. Some researchers have argued the replication crisis is not a true crisis because of methodological differences between the original studies and the replications (Gilbert, King, Pettigrew, \& Wilson, 2016; Van Bavel, Mende-Siedlecki, Brady, \& Reinero, 2016) or that many of the effects replicated the original studies but evidence was weak in both cases anyway (Etz \& Vandekerckhove, 2016). Others have proposed modifications to the accepted practices by which 
researchers conduct their science (Simmons et al., 2011). Modification to the rules include methodological decisions (such as when to stop data collection) and analysis decisions (such as stricter requirements on deciding which outliers to exclude or which factors to include in an analysis).

Another response has been a proposal to change the criterion for asserting that an effect is significant. It was suggested that simply altering the p-value is a straightforward step that could improve the reproducibility of research by increasing statistical standards (Benjamin et al., 2017). The specific suggestion in this paper was to change the cut-off for determining the significance for a new effect from $p<.05$ to $p<.005$. Changing the criterion for significance in this way would certainly reduce the number of studies that could not be replicated, but what other consequences would this change have? Using data simulations, the following question was explored: What is the best criterion for statistical significance? An ideal criterion for statistical significance is one that perfectly discriminates between real effects and null effects. One analytic technique that is intended to measure the discriminability of a test is signal detection theory (Green \& Swets, 1966). Although signal detection theory has been applied widely to other kinds of tests, it has not yet been applied to the current debates regarding criteria for statistical significance.

Signal detection analysis involves categorizing outcomes into four categories. Applied to criteria for statistical significance, a hit occurs when there is a true effect and the analysis correctly identifies it as significant (see Table 1). A miss occurs when there is a true effect but the analysis identifies it as not significant. A correct rejection occurs when there is no effect and the analysis correctly identifies it as not significant, and a false alarm occurs when there is no effect but the analysis identifies it as significant. In statistics, Type I errors (false alarms) and Type II errors (misses) are sometimes considered separately, with Type I errors being a function of the alpha level and Type II errors being a function of power. An advantage of signal detection theory is that it combines Type I and Type II errors into a single analysis. 
The goal of the current paper is twofold. One goal is apply signal detection theory techniques to issues concerning the criteria for statistical significance. Signal detection theory allows the separate assessment of the discriminability of a measure (e.g. p-values) and also the bias for any given criterion (e.g. $p<.005$ ). The second goal is to highlight important issues regarding the interpretation of statistical measures as evidence for an effect to a broader audience of psychology researchers by producing graphs of (simulated) data. The idea is that plots of data might improve comprehension of these issues compared with mathematical equations.

Table 1. Signal detection classification of data based on the example criteria $p<.05$ (left) and Bayes factor (BF) $>3$ (right) for a true effect (Cohen's $d=0.50$ ) and a null effect (Cohen's $d=0$ ).

\begin{tabular}{|c|c|c|c|c|c|}
\hline & $\begin{array}{c}P<.05 \\
\text { "Significant" }\end{array}$ & $\begin{array}{c}\text { P > .05 } \\
\text { "Not Sig" }\end{array}$ & & $\begin{array}{c}\mathrm{BF}>3 \\
" \mathrm{H}_{1}>\mathrm{H}_{0} "\end{array}$ & $\begin{array}{l}\mathrm{BF}<1 / 3 \\
" \mathrm{H}_{0}>\mathrm{H}_{1} "\end{array}$ \\
\hline$d=.50$ & Hit & Miss & \multirow{2}{*}{$\begin{array}{c}d=.50 \\
d=0\end{array}$} & Hit & Miss \\
\hline$d=0$ & False Alarm & $\begin{array}{c}\text { Correct } \\
\text { Rejection }\end{array}$ & & $\begin{array}{l}\text { False } \\
\text { Alarm }\end{array}$ & $\begin{array}{l}\text { Correct } \\
\text { Rejection }\end{array}$ \\
\hline
\end{tabular}

\section{Data Simulations}

Data were simulated for two independent groups of 64 participants each, which corresponds to $80 \%$ power at an alpha level of .05 for a two-tailed independent-samples t-test. Data for one group was sampled from a normal distribution with a mean of $50 \%$ correct and a standard deviation of $10 \%$ (such as might be found on a memory test). The data for the other group was sampled from a normal distribution with a mean of $50 \%$ (for studies with a null effect) or $45 \%$ (for studies with an effect size of Cohen's $d=.50$ ) and a standard deviation of $10 \%$. The data were submitted to an independent-samples t-test (all simulations and analyses were conducted in R; R Core Team, 2017). Details of the simulation are available in the on-line supplementary materials.

Data were simulated from 20 studies, half of which had an effect size of 0 and half had a medium effect size (Cohen's $d=.50$ ). The result from each simulated study was classified as a hit or 
miss (for studies modeled as a medium effect) or as a correct rejection or false alarm (for studies modeled as a null effect). The classification was based on four criteria for statistical significant related to p-values: $p<.10, p<.05, p<.005$, and $p<.001$. The outcomes across all 20 studies were summarized into a hit rate, a miss rate, a correct rejection rate, and a false alarm rate for each criterion. This process was repeated 100 times. The means across all 100 sets of 20 studies are shown in the left side of Figure 1. The receiver operator characteristic $(\mathrm{ROC})$ curve plots hits as a function a false alarms. These are plotted for one set of 20 studies and averaged across all 100 sets of 20 studies in Figure 2. Bayes factors, which are also plotted, will be discussed later.

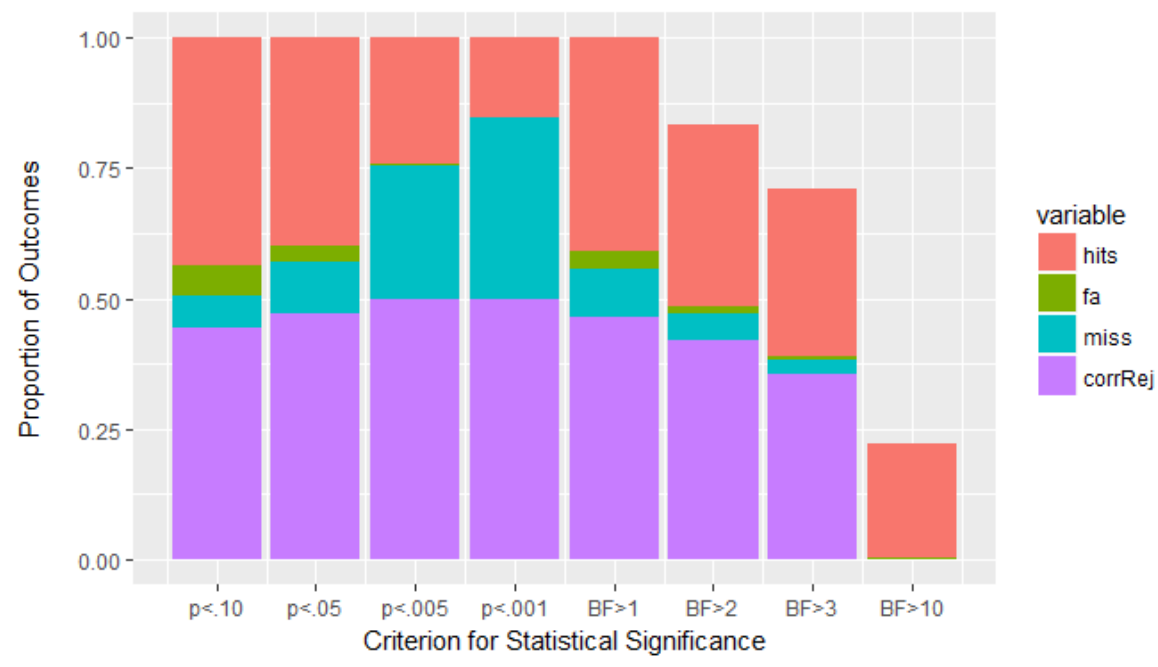

Figure 1. Proportion of each outcome as a function of the criterion for significance. For criteria of Bayes factors greater than 2, 3, or 10, studies that produced a Bayes factor less than the criterion but greater than the inverse of the criterion are considered inconclusive, which is why the total proportion of outcomes does not equal 1. 


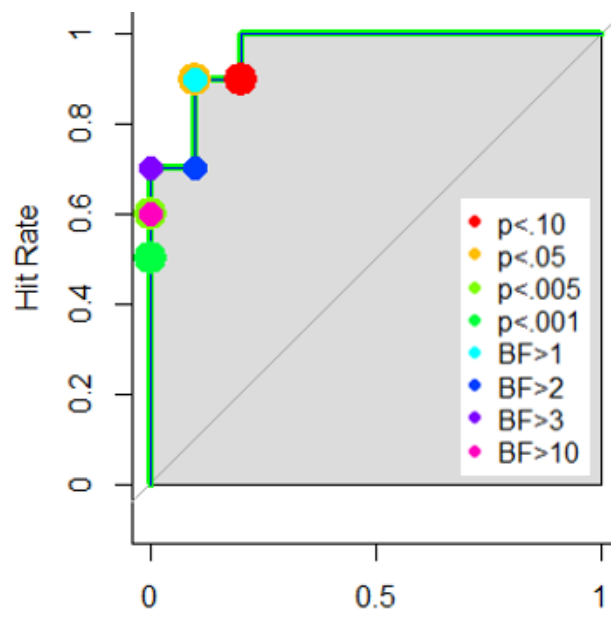

False Alarm Rate

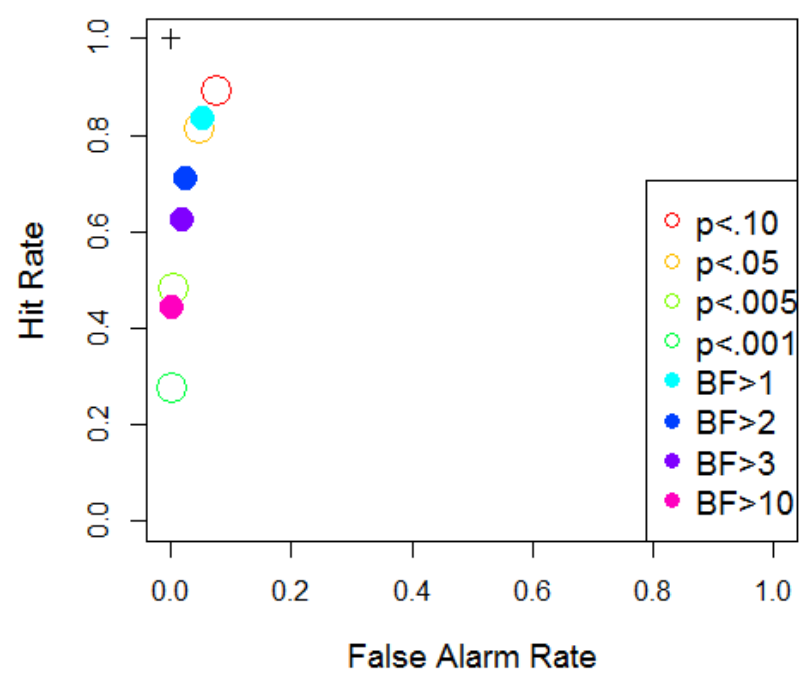

Figure 2. Mean hit rates are plotted as a function of mean false alarm rates and the criterion for significance for one set of 20 studies (left panel) and all 100 sets of 20 studies (right panel). In the left panel, receiver operator characteristic (ROC) curves are plotted for criteria based on $p$ values (thick green line) and Bayes factor (thin blue line) for one set of 20 studies. The two lines are identical (as was the case for all 100 sets of 20 studies). Area under the curve (AUC) is the shaded area. In the right panel, the black cross is positioned at optimal performance $(100 \%$ hit rate and $0 \%$ false alarm rate).

In selecting a criterion for statistical significance, researchers must select a measure (e.g. pvalues) and a cut-off within that measure (e.g. .05). A measure can be evaluated by assessing its ability to discriminate between real and null effects. Discriminability can be quantified by calculating the area under the ROC curve (AUC; Macmillan \& Creelman, 2008). With respect to evaluating cut-offs for a specific measure (e.g. comparing .005 to .05 ), we can calculate the location of each cut-off on the ROC curve. Location on the curve is a measure of bias. We will consider to each of these measures in turn.

\section{Analysis of p-value Discriminability}

To measure discriminability of p-values, the AUC was computed 100 times, once for each set of 20 studies. Unlike the discriminability measure of $d^{\prime}$, the discriminability measure of AUC makes no assumptions regarding the underlying distributions, which is critical because distributions of $p$-values are not normally distributed. Higher AUCs indicate better ability to discriminate real effects from null 
effects. If discrimination were perfect, the curve would follow the left and top boundaries in Figure 2, and the AUC would equal 1 (i.e. the entire area would be under the curve). If discrimination were at chance, the curve would follow the diagonal line in Figure 2, and the AUC would be .5 (i.e. only $50 \%$ of the area would be under the curve). As is apparent in Figure 2, p-values produced curves that were closer to 1 (perfect performance) than to .5 (chance performance). The mean AUC was .96 (median = $.97, \mathrm{SD}=.04)$. Thus, $\mathrm{p}$-values are effective but not perfect at discriminating between real and null effects. As a tool for discrimination, the p-value performed well under these circumstances. The use of $p$-values as a tool to determine statistical significance has been challenged since its inception, and the criticism has recently intensified due to seemingly large-scaled failures to replicate studies published in top journals. But an AUC of $.96-.97$ suggests respectable discriminability performance. As a point of comparison, the AUCs for detecting breast cancer from digital or film mammography are .78 and .74, respectively (Pisano et al., 2005). Even though p-values produce high discriminability, it is not perfect in this scenario, which means that errors will be made. It also means that there will be ways to exasperate these errors, which can lead to increased false alarm rates. However, given that discriminability is so high, it seems that $p$-values provide good value in discriminating between real and null effects, so alternative methods to reduce false alarm rates rather than eliminate p-values altogether (e.g. Trafimow \& Marks, 2015) might be appropriate.

\section{Analysis of $p$-value Bias}

Signal detection theory distinguishes between discriminability and bias. As applied to the case of criteria for statistical significance, discriminability refers to the criterion's performance at identifying real effects versus null effects, and bias refers to whether the errors tend to be false alarms or misses. Assessing bias can be useful for selecting the appropriate criterion for asserting statistical significance. For example, assume that the cost of a miss is equivalent to the cost of a false alarm in a particular field. 
In that case, optimal utility would be achieved by setting the criterion in such a way that its point on the receiver operator characteristics (ROC) curve is the one that falls closest to the upper left corner in Figure 2. The Euclidean distance between each point on the ROC curve and the point of perfect performance is plotted in Figure 3. For the scenario that was simulated, an alpha level of .10 would come closer to achieving that maximum-utility outcome than an alpha level of .005 . Lowering the criterion for statistical significance to $p<.005$ would increase the number of studies that will replicate by decreasing false alarms, but it would do so at the cost of missing real effects. Note the proportion of misses in Figure 1 across the various criteria, particularly for the criterion of $p<.005$. Misses are bad for science. Assuming that null effects are theoretically interesting and practically important, it is important to determine which null effects are due to a genuine lack of difference versus a miss of a true effect. Is the trade-off to increase replicability worth the large increase in misses? Perhaps science can adopt alternative means to improve replicability without sacrificing so many missed hits, such as minimizing incentives for publishing significant findings only and publishing null results.

\section{$\mathrm{N}=64 \mathrm{~d}=0.5$}

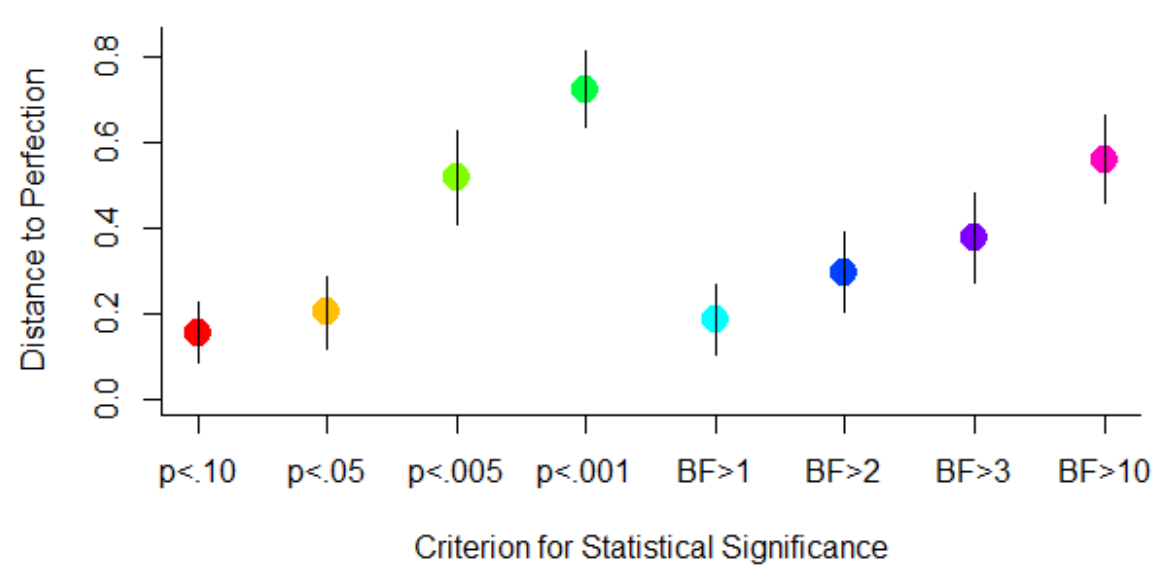

Figure 3. Distance to perfection was calculated as the Euclidean distance between each point on the ROC curve (see Figure 2) and the top-left corner (which corresponds to $100 \%$ hit rate and $0 \%$ false alarm rate) across all 100 sets of 20 studies. A lower distance to perfection score indicates better discriminability between real and null effects. Error bars represent $95 \%$ confidence intervals. 


\section{Bayes Factors Versus p-values}

An alternative to $p$-values is to use Bayes factors. Bayes factor refers to the likelihood that the alternative hypothesis is true relative to the null hypothesis given the data. A Bayes factor of 1 corresponds to equal likelihood for the alternative and the null hypotheses, and a Bayes factor greater than 1 is evidence for the alternative hypothesis relative to the null hypothesis. Bayes factors quantify how well a hypothesis predicts the data relative to a competing hypothesis (such as the null hypothesis), and thus is a continuous measure. However, Bayes factors between 1-3 are considered weak or anecdotal evidence, so a Bayes factor of 3 is akin to a criterion for significance, although some prefer a Bayes factor of 10 or greater (see Table 2). To measure discriminability and bias for Bayes factors, the simulated studies were also evaluated for statistical significance using four criteria related to Bayes factor (BF): $\mathrm{BF}>1, \mathrm{BF}>2, \mathrm{BF}>3$, and $\mathrm{BF}>10$. The BayesFactor R package (Morey, Rouder, \& Jamil, 2014) was used to calculate the Bayes factors.

Table 2. Overview of relationship between Bayes factor and conclusion about the evidence being in favor of the alternative hypothesis $\left(\mathrm{H}_{A}\right)$ or the null hypothesis $\left(\mathrm{H}_{0}\right)$. Adapted from Wetzels et al. (2011) and Jeffreys (1961).

\begin{tabular}{|l|l|}
\hline Bayes factor & Interpretation \\
\hline$>100$ & Decisive evidence for $\mathrm{H}_{A}$ over $\mathrm{H}_{0}$ \\
\hline $30-100$ & Very strong evidence for $\mathrm{H}_{A}$ over $\mathrm{H}_{0}$ \\
\hline $10-30$ & Strong evidence for $\mathrm{H}_{\mathrm{A}}$ over $\mathrm{H}_{0}$ \\
\hline $3-10$ & Substantial evidence for $\mathrm{H}_{\mathrm{A}}$ over $\mathrm{H}_{0}$ \\
\hline $1-3$ & Anecdotal evidence for $\mathrm{H}_{A}$ over $\mathrm{H}_{0}$ \\
\hline 1 & No evidence \\
\hline $1 / 3-1$ & Anecdotal evidence for $\mathrm{H}_{0}$ over $\mathrm{H}_{\mathrm{A}}$ \\
\hline $1 / 10-1 / 3$ & Substantial evidence for $\mathrm{H}_{0}$ over $\mathrm{H}_{A}$ \\
\hline $1 / 30-1 / 10$ & Strong evidence for $\mathrm{H}_{0}$ over $\mathrm{H}_{A}$ \\
\hline $1 / 100-1 / 10$ & Very strong evidence for $\mathrm{H}_{0}$ over $\mathrm{H}_{A}$ \\
\hline$<1 / 100$ & Decisive evidence for $\mathrm{H}_{0}$ over $\mathrm{H}_{A}$ \\
\hline
\end{tabular}

As shown in Figure 2, the AUCs related to Bayes factor were also quite high. In fact, the AUCs for Bayes factor corresponded perfectly to the AUCs for $p$-values. This means that for the situation 
simulated here, Bayes factors are not any better (or worse) than p-values at discriminating real effects from null effects. In other words, Bayes factor incurs no advantage over $p$-values at detecting a real effect versus a null effect for the current scenario. This is because Bayes factors are redundant with $p$ values for a given sample size. In these simulations, there was a near-perfect linear relationship between the (log of the) Bayes factors and the (log of the) $p$-values.

Although the discriminability between $p$-values and Bayes factors was equivalent across a variety of situations, as revealed by equal AUCs (see Figure 4a, for example), the exact relationship between them differs as a function of sample size. For 30 different sample sizes ranging from 32 to 2000 per group, 100 simulations of 20 studies were conducted (10 with a Cohen's d modeled at .50 and 10 with a Cohen's d modeled at 0). For each sample size, a linear regression was conducted to predict the log of the Bayes factor from the log of the p-value. The results are shown in Figure 4. These simulations show near-complete redundancy between $p$-values and Bayes factors. This redundancy also supports the conclusion that for the conditions simulated, p-values and Bayes factors are equally adept at distinguishing real effects from null effects. 
a

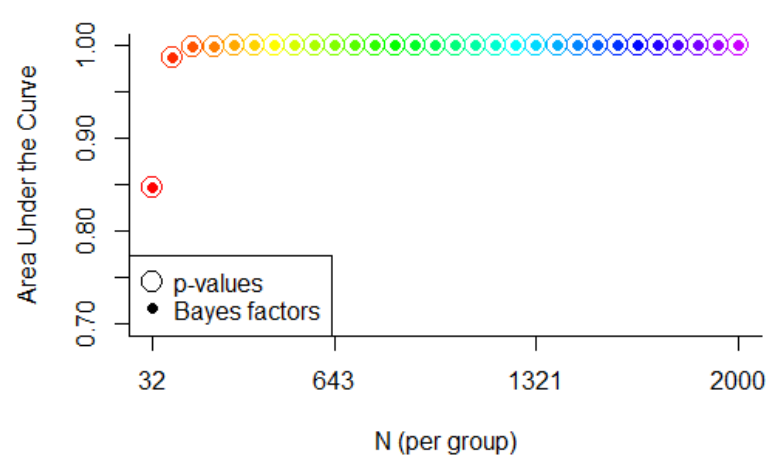

b

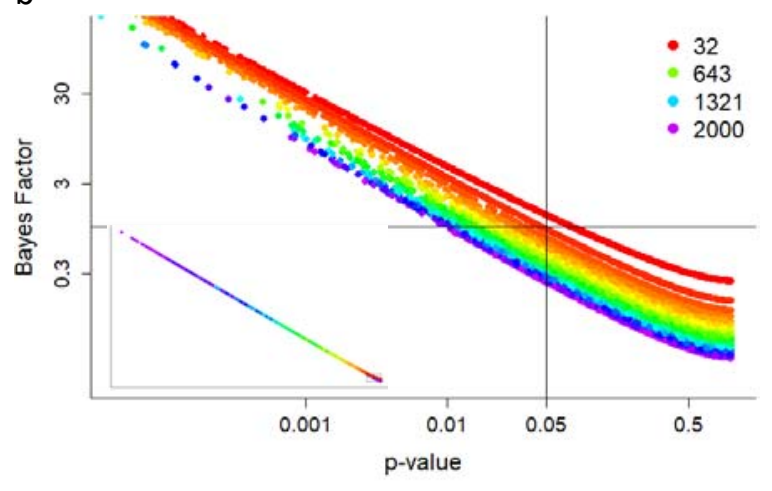

C

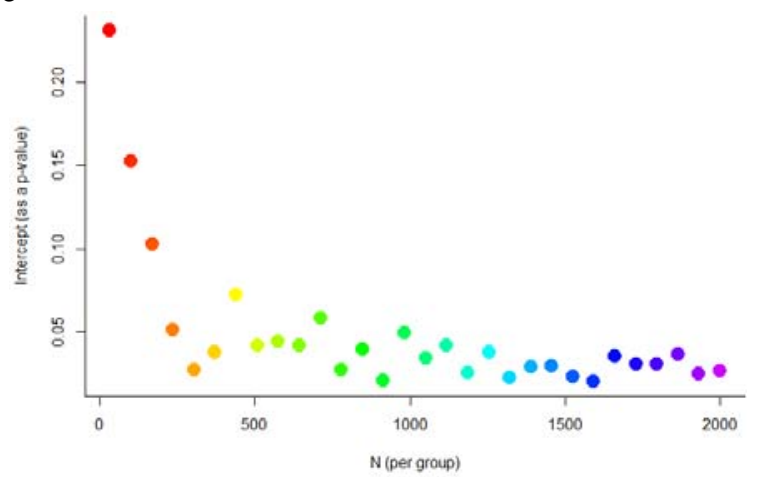

d

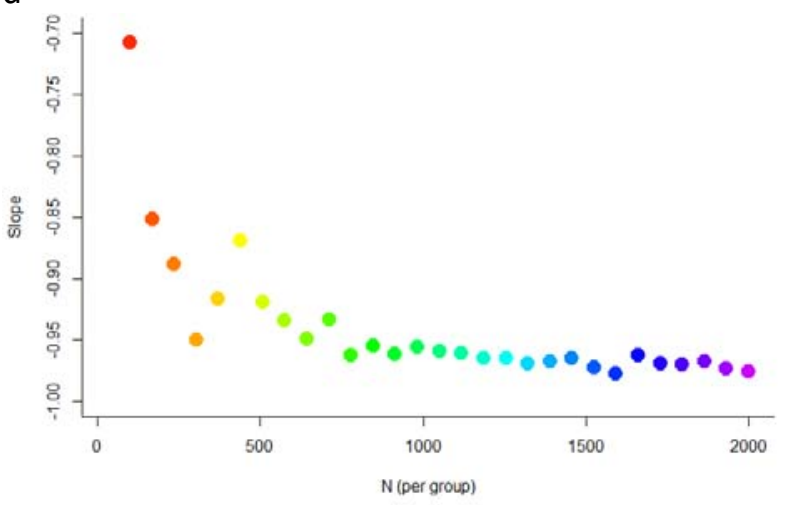

Figure 4. Outcomes from 1000 studies simulated as a null effect and 1000 studies simulated as a medium effect (Cohen's $d=.50$ ) for each of 30 different sample sizes ranging from 32 to 2000 . Color corresponds to sample size. Panel a shows the area under the curve (AUC) for $p$-values and Bayes factors as a function of sample size. A bigger AUC indicates better discrimination between real and null effects. Panel $b$ shows the relationship between $p$-value and Bayes in the range for which $p$-values are highest (the inset shows the relationship for the entire range, and the dotted box shows the area that has been expanded in the main figure). The legend shows 4 examples of sample sizes. The black vertical line corresponds to a p-value of .05, and the black horizontal line corresponds to a Bayes factor of 1 . Panels $\mathrm{c}$ and $\mathrm{d}$ show the intercepts and slopes from linear regressions that predict the log of the Bayes factor from the log of the $p$-values. The intercept is the $p$-value that corresponds to a Bayes factor of 1 , so it corresponds to the value of the $p$-value along the horizontal line in panel $b$. The slope, plotted in panel $d$, corresponds to the steepness of the curves in panel $b$.

Despite equivalence in discriminability between p-values and Bayes factor, these simulations reveal a previously acknowledged discrepancy in the conclusions supported by the two types of criteria (Lindley, 1957). Specifically, in Figure 4b, all data points on or to the left of the black vertical line that are also below the black horizontal line would be classified as significant according to the criterion of $p<$ 
.05 but according to a Bayes factor interpretation, the evidence would favor the null hypothesis over the alternative. This illustrates why it is possible to get results for which the p-value indicates a significant finding (i.e. evidence for the alternative hypothesis) but the Bayes factor shows evidence for the null hypothesis relative to the alternative. These conflicting outcomes occurred in studies for which sample size (or, more precisely, power) was high. These simulations help illustrate the point that for highpowered studies, a p-value of .05 is more evidence for the null hypothesis than for the alternative hypothesis.

The difference in the value of the intercept as sample size increases provides one way to visualize this aspect of the relationship between $p$-values and Bayes factors (see Figure 4c). The intercept corresponds to the point at which Bayes factor equals 1, which corresponds to the point at which there is equal evidence for the alternative and null hypotheses. That intercepts decreased as sample size increased indicates a Bayes factor of 1 corresponds to an increasingly smaller p-value as sample size increases. In other words, to get a Bayes factor greater than 1 (evidence in favor of the alternative relative to the null hypothesis), a smaller $p$-value is needed as sample size increases.

Given that the same p-value (e.g. .05) leads to a range of Bayes factors that can have different interpretations (e.g. evidence for the alternative hypothesis in cases of lower power and evidence for the null hypothesis in cases of higher power), one conclusion that can be highlighted by these simulations is that $p$-values should only be interpreted in the context of the power of the study. This point dates back to Neyman and Pearson, but bears repeating as sometimes researchers incorrectly interpret $p$-values without consideration of power, such as when a high-powered study produces a pvalue that is slightly less than .05 and is then interpreted as a significant effect.

Despite equivalent discriminability across $p$-values and Bayes factors, one advantage previously pointed out for Bayes factors over $p$-values is that statistical significance cannot be inferred based on $p$ values alone without also considering statistical power (Jefferys, 1990). To make inferences about 
power, one must know the true effect size. Given publication bias to publish only significant findings, the published literature is likely to overestimate effect size (Ioannidis, 2005), making it difficult to properly estimate true effect sizes. This point can be illustrated in a number of ways (e.g. loannidis, 2005). Here, this point will be illustrated again using simulations of 1000 studies with a true effect size of $d=.50$ and 1000 studies with a true effect size of $d=0$. The measured effect size across all of the studies in a set converges to the true effect size, but the effect size that would be reported in the literature given the standard expectation of $p<.05$ overestimates the true effect size, particularly for low powered studies (see Figure 5). Note that the overestimation of effect size due to the cut-off of $p<$ .05 is an insufficient reason to reject p-values or the cut-off of .05 . Rather, it is reason for the field to develop avenues by which to publish non-significant effects so that the literature reflects the true effect size.

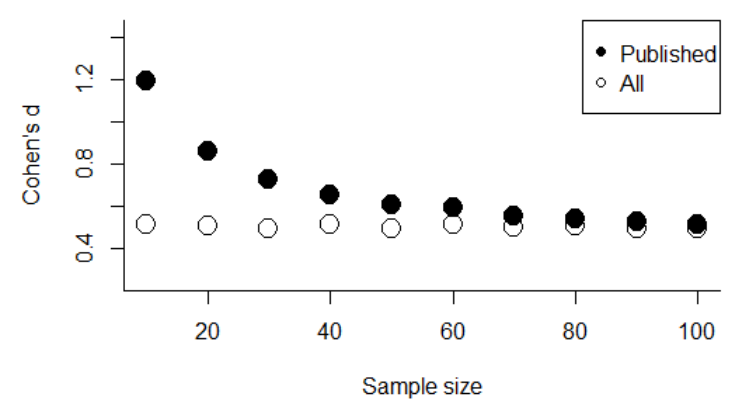

Figure 5. Mean measured effect size, as Cohen's d, as a function of sample size across all 1000 studies (open circles) and for studies that would meet the standard publication criterion of $p<$ .05. Simulations were done with Cohen's $d$ set to .50 . Note that when averaged across all studies, the measured Cohen's d matches the true (i.e. simulated) Cohen's d.

That publication bias leads to overestimation of effect size is relevant for the debate regarding p-values verses Bayes factors. Effect size must be known to estimate power, and power must be known to interpret $p$-values, specifically $p$-values great than .01 . But it is difficult to estimate effect size when publication bias leads to inflation of effect size in the literature. Indeed, one could account for the failure to replicate $53 \%$ of 100 studies in psychology (cf. Open Science Collaboration, 2015) based solely 
on knowledge of that publication bias produces overestimation of effect size, which means that the replications did not achieve the power they claimed to have achieved. For example, if the true effect size had been $d=.5$, but publication bias reported the effect size to be $d=.7$, the sample needed to achieve $80 \%$ power for $d=.7(N=33)$ would only achieve $52 \%$ power for an effect size of $d=.5$. This means that if all the studies had been true effects of $d=.5$ but reported effect size of $d=.7$ and all replications had achieved $80 \%$ power based on the reported effect size, only $52 \%$ would have produced significant effects. In contrast, interpreting Bayes factors do not depend as much on knowing power as does interpreting $\mathrm{p}$-values. This confers an advantage of Bayes factors over $\mathrm{p}$-values.

The slopes between p-values and Bayes factors also decreased as sample size increased. This simply illustrates that when sample size is small, strong evidence from the Bayes factor for either the alternative hypothesis or for the null hypothesis is improbable, whereas the full range of $p$-values is still possible. This illustrates another advantage of Bayes factors over $p$-values. When there is less evidence (in this case, due to a smaller sample size), there is more uncertainty in the conclusion. This uncertainty is captured by better by Bayes factors than p-values, particularly for null effects. Consider the distribution of $p$-values and Bayes factors across a range of under-powered to appropriate powered studies (see Figure 6). When there is a true effect (simulations for which Cohen's d was modeled as .5), both $p$-values and Bayes factors led to stronger inferences for the alternative over the null hypothesis as sample size increases. For true effects, $p$-values and Bayes factors both performed well. But for null effects (simulations for which Cohen's d was modeled as 0), Bayes factors better converged on evidence for the null hypothesis over the alternative hypothesis as sample size increased. The distribution of $p$ values was uniform regardless of sample size. Given the linear relationship between p-values and Bayes factors, one could interpret high p-values as evidence for the null hypothesis, but to do so would necessarily depend on knowing power. For example, a p-value of .40 would be equally likely for studies on a null effect that have incredibly low power as that have high power. In contrast, lower Bayes factors 
are more likely as power increases, and Bayes factors less than .20 are unlikely to be achieved without at least $80 \%$ power.
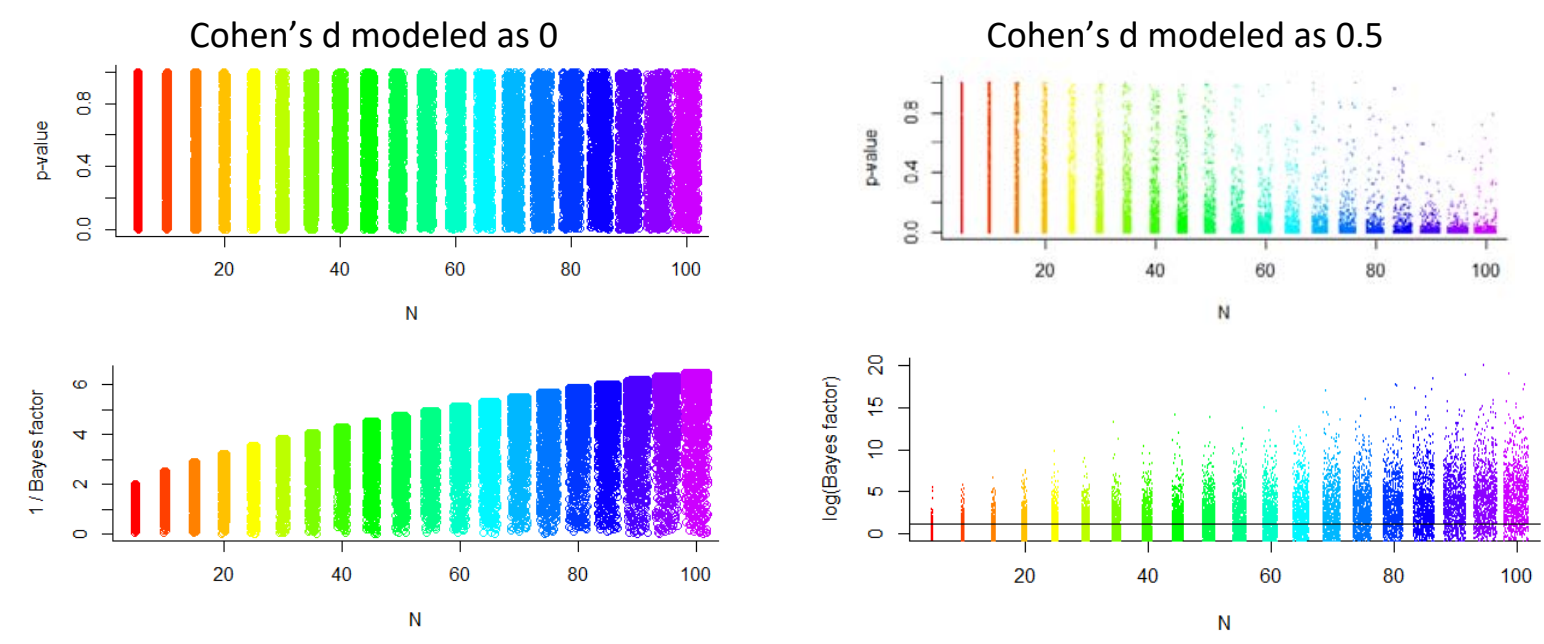

Figure 6. Distribution of $p$-values (top row) and Bayes factors (bottom row) as a function of sample size and whether the simulations were done with Cohen's $d$ modeled as 0 (left column) or as 0.5 (right column). Each point corresponds to a single study. In the top left ( $p$-values for null effects), the distributions are uniform across sample size, thereby showing insensitivity in $p$ values to power. In contrast, in all other plots, the distributions change as a function of sample size. Not that in the bottom right graph, the inverse of the Bayes factor is plotted, so a higher value corresponds to increased evidence for the null hypothesis over the alternative hypothesis. In the bottom right corner, the log of the Bayes factor is plotted in order to better visualize across all values including extremely high values. Here, a higher value correspond to increased evidence for the alternative hypothesis over the null hypothesis.

One of the reasons that has been offered as motivation to use Bayes factors is that they can provide evidence for the null hypothesis (e.g. Kass \& Raftery, 1995; Stern, 2016). In contrast, a p-value less than some criterion (such as .05) suggests that the null hypothesis can be rejected, and a p-value greater than that criterion implies a failure to reject the null hypothesis, which is decidedly different from accepting the null hypothesis. However, given the near-linear relationship between $p$-values and Bayes factors, higher $\mathrm{p}$-values can be interpreted as evidence for the null hypothesis. Assuming there is information in the Bayes factors, there is also information in the specific magnitude of the $p$-value. This means that scientists should report exact $p$-values, even for those deemed not significant. However, the strength of the evidence depends on knowing power, which depends on knowing the true effect size, 
which can be problematic, as discussed above. Furthermore, Bayes factors are more intuitive to interpret than p-values given that they provide how much more likely one hypothesis is over another. The intuitive appeal of Bayes factors and the fact that their interpretation does not depend on knowing the true effect size are arguably reasons to use Bayes factors rather than p-values. It is unreasonable, however, to argue that Bayes factors allow better, or even different, interpretations than $p$-values. For the conditions simulated here, the two are equivalent in their evidentiary discriminability, as measured with AUCs.

\section{Including Priors}

Whereas Bayes factors do not take into account the prior odds of an effect being real, the posterior odds do. Posterior odds can be calculated by multiplying the Bayes factor by the prior odds (see Equation 1). Posterior odds are the probability of the alternative hypothesis $\left(M=H_{1}\right)$ given the data D over the null hypothesis $\left(M=H_{0}\right)$ given the data $(D)$. The discriminability of the posterior odds, as measured with AUC, is also the same as the discriminability of the Bayes factors and p-values (see Figure 7). Adding information about prior odds to the Bayes factor merely shifts the points along the ROC curve but does not alter discriminability.

$$
\begin{array}{ll}
\text { Posterior odds } & \begin{array}{l}
\text { Bayes factor } \\
p\left(M=H_{1} \mid D\right)
\end{array}=\frac{p\left(D \mid M=H_{1}\right)}{p\left(D=H_{0} \mid D\right)} \times \frac{p\left(M=H_{1}\right)}{p\left(M=H_{0}\right)}
\end{array}
$$



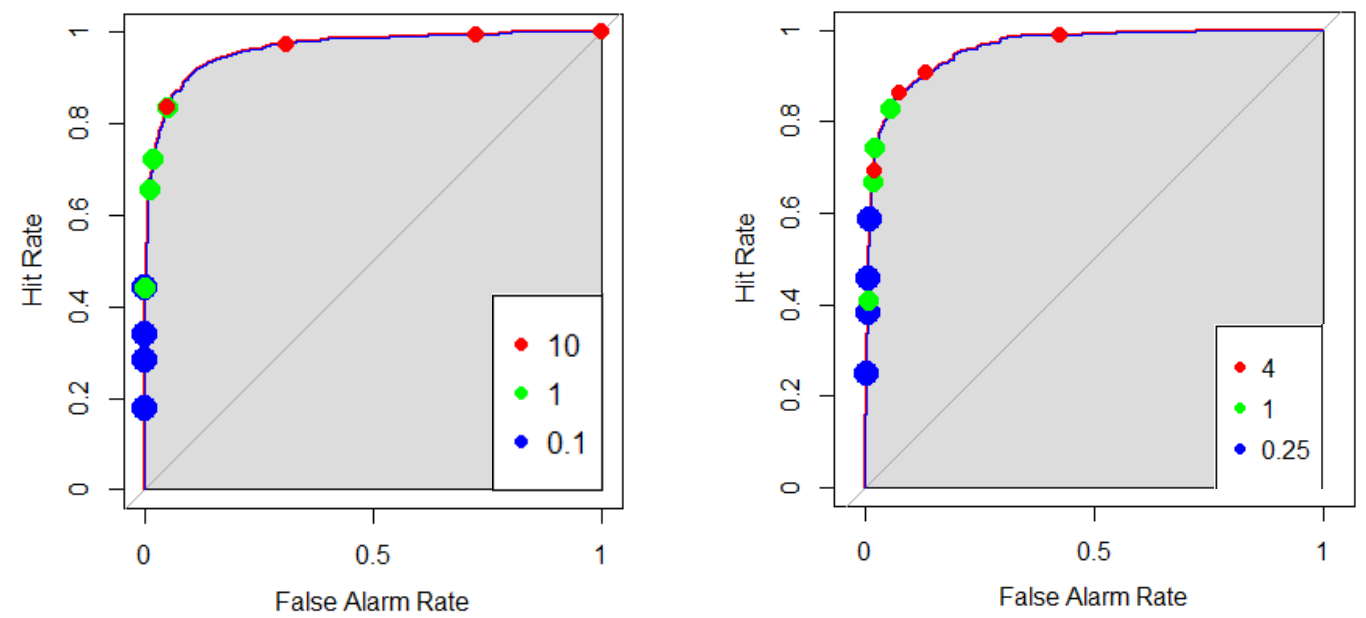

Figure 7. Hit rates are plotted as a function of false alarm rates and criterion for significance for 100 sets of 20 studies (left panel) and 100 sets of 15 studies (right panel). Each point corresponds to a different criterion for significance related to the posterior odds $(1,2,3$, and 10 , not labeled but for each cluster of 4 , the points go sequentially from top-right corner to bottomleft corner) as a function of the prior odds (see legend). In the left panel, null effects ( $d=0 ; 10$ studies per set) were modeled as often as real effects ( $d=.5 ; 10$ studies per set). In the right panel, null effects ( $d=0 ; 12$ studies per set) were modeled at a ratio of 4 to 1 compared with real effects ( $d=.5 ; 3$ studies per set). In each study, there were 64 participants per group. The receiver operator characteristic (ROC) curves are plotted for three different sets of prior odds for each panel. The area under the curve (AUC) is shown in grey. The curves and AUCs are identical across all prior odds in each panel.

\section{Questionable Research Practices}

Surprisingly, this story of high discriminability for both p-values and Bayes factors was unaffected by the use of questionable research practices such as an optional stopping rule for data collection. Questionable research practices have been identified as particularly problematic and the first recommended change in practices is to designate the number of participants to be run ahead of time, rather than using an optional stopping rule (Simmons et al., 2011). In a new set of simulations, each simulated study was conducted with 30 participants per group with either a Cohen's $d=.50$ or $d=0$. A lower sample size was used given that published studies tend to be under-powered. For each effect size, 10 studies were simulated, and this was repeated this 100 times. To try to mimic a typical form of the optional stopping rule, for each study, if the p-value was between .20 and .05 , an additional 10 
participants were added per group. After this addition, if the $p$-value was less than .05 , data collection stopped; otherwise the process was repeated up to 9 more times. The original 30 per group were then assessed using Bayes factor. If the original group led to a Bayes factor between 1 and 3, 10 more participants were added until the Bayes factor was greater than 3 or until 100 more participants had been added. The optional stopping rule produced differences in the AUCs relative to the original sample, but the differences were not systematic. Sometimes running additional unplanned participants improved discriminability and other times it worsened discriminability (see Figure 8 ). The decision to add more participants occurred more frequently for $p$-values $(M=20 \%, S D=9 \%)$ than for Bayes factors $(M=16 \%, S D=8 \%)$, but the increased frequency did not lead to better or worse discrimination overall for $p$-values versus Bayes factors. Unreported simulations showed that other versions of questionable research practices such as flexibility in choosing outliers to include or running multiple tests (e.g. with equal and unequal variances) and selecting the lowest $p$-value or highest Bayes factor also produced equivalent AUCs across p-values and Bayes factors. 
a

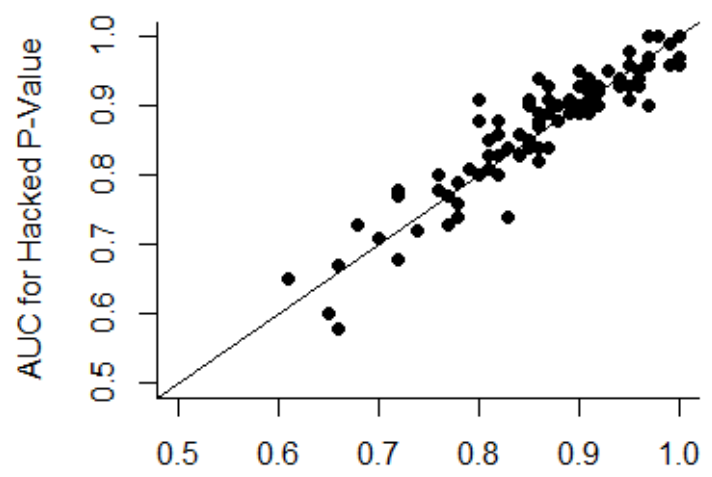

Area Under Curve for P-Value

C

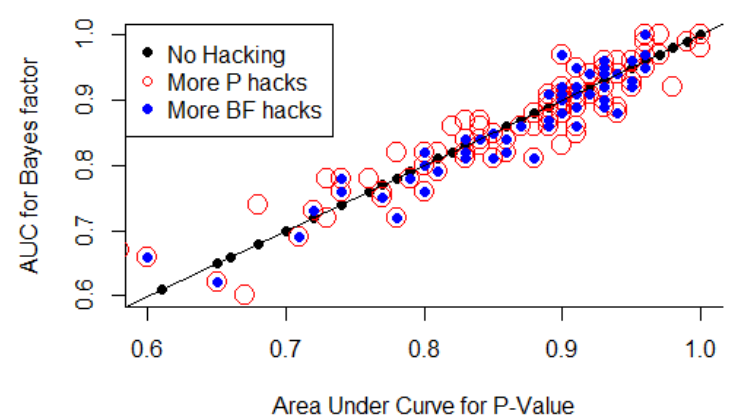

b

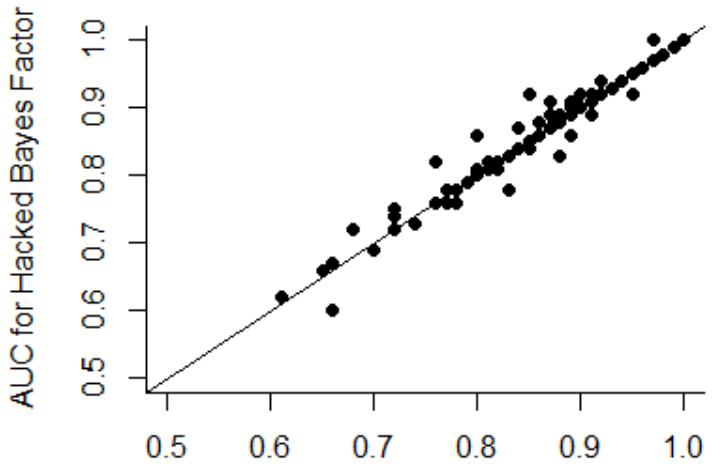

Area Under Curve for Bayes Factor

d

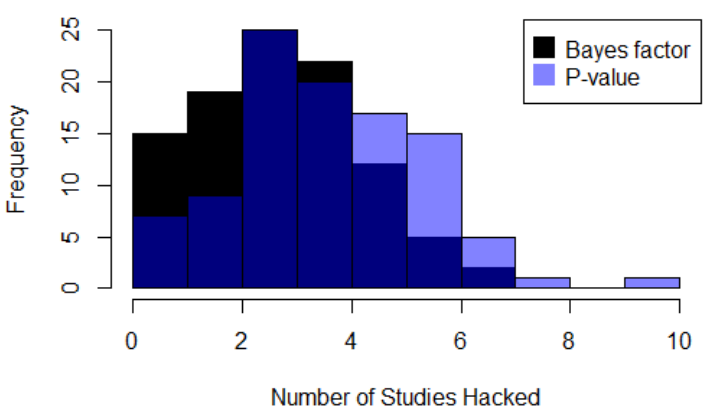

Figure 8. The area under the curve (AUC) for hacked studies versus the original studies are plotted for $p$-values (panel a) and Bayes factors (panel b). The AUCs for Bayes factors are plotted as a function of $p$-values and whether the study was hacked in panel c. A higher AUC indicates better discrimination between real and null effects. The black circles represent analyses on the original 30 data points. The red and blue circles represent simulations for which additional subjects were added to try to get a $p$-value less than .05 ( $p$-hacking) and/or a Bayes factor greater than 3 (bfhacking). Blue circles are from simulations for which there were more instances of $p$-hacking than bf-hacking, and red circles are from simulations for which there were equal or more instances of bf-hacking than $p$-hacking. Panel $d$ shows a frequency plot across the 100 simulations of the number of times within each set of 20 studies that there was p-hacking (blue) or bf-hacking (black).

How can these questionable research practices have no impact the discriminability of real

effects from null effects? The reason is that these questionable research practices increase the false alarm rate but they also increase the hit rate (see Figure 9). Much of the attention on the replication crisis has sought to minimize false alarms, but it is also necessary to discuss the corresponding increase in the number of misses (i.e. the decrease in the number of hits). Discriminability between real effects 
and null effects takes into account both the false alarm rate and the hit rate. A decreased hit rate directly corresponds to an increased miss rate. Even when studying a real effect, the effect will not be detected as often when not using multiple tests or optional stopping rules. Thus, reducing these questionable research practices will have the positive effect of decreasing false alarm rates (see also loannidis, 2005), but will also have the negative effect of increasing miss rates.

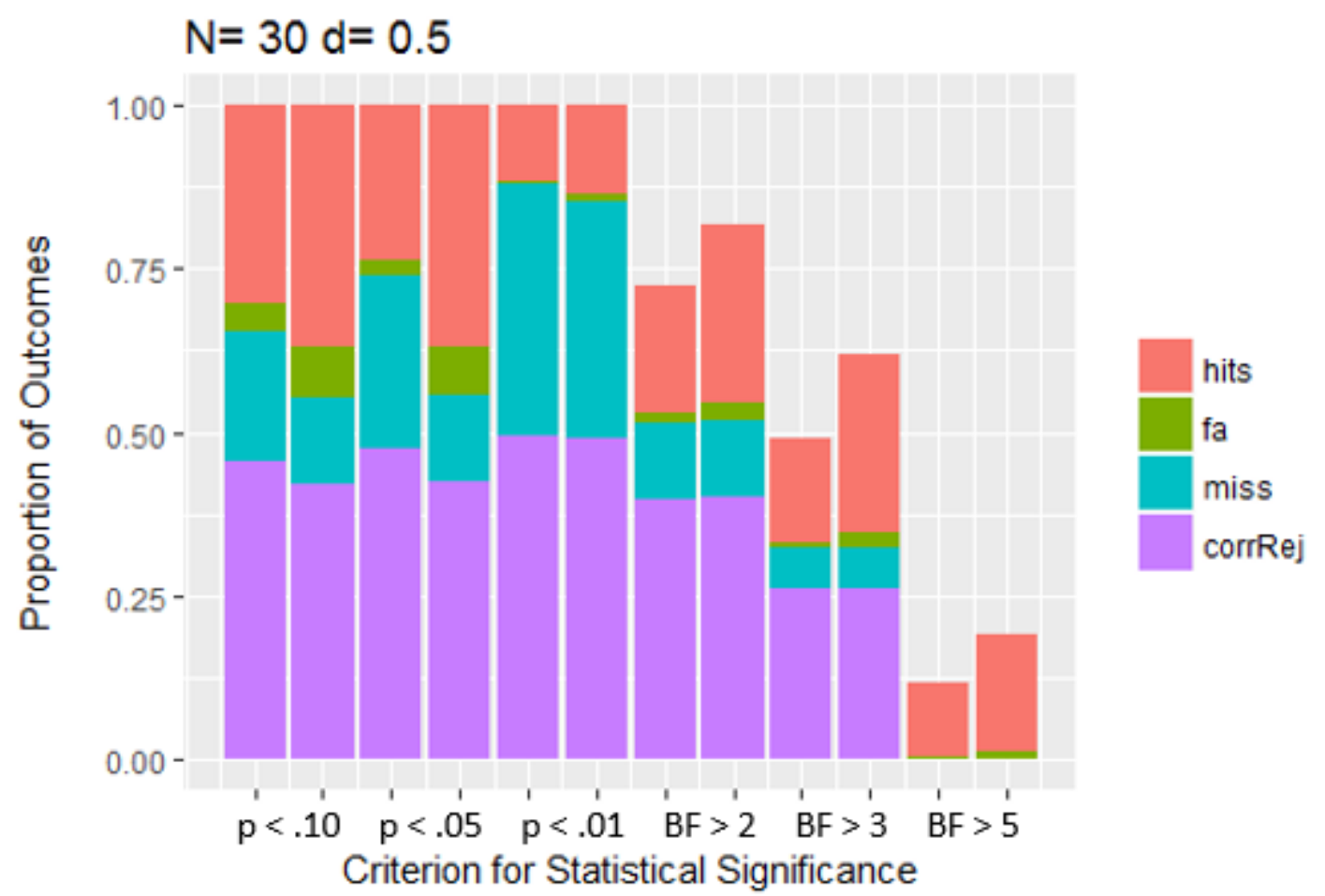

Figure 9. Proportion of hits, false alarms (fa), misses, and correct rejections (corrRej) as a function of criteria for statistical significance and whether or not the study involved $p$-hacking or bf-hacking, in the form of an optional stopping rule. In each set of two bars, the one on the left is for the original 30 participants and the bar on the right is for the hacked condition for which up to 100 participants could have been added. For each set of two bars, note that there is an increase in both the false alarm rate (green portion) and the hit rate (red portion) when an optional stopping rule was used.

\section{Bayes Factors and Bias}

As with p-values, we can consider bias related to Bayes factors. As shown in Figure 3 , the cutoffs that achieves maximize utility assuming equal weights given to false alarms and misses is Bayes 
factor $>1$. This contrasts with the typical interpretation of Bayes factor (e.g. Table 2) for which Bayes factors between 1-3 are considered anecdotal evidence.

Unlike with p-values, the threshold that should be used for Bayes factors does not vary as much as do $p$-values with changes in sample size (see Figure 10). Compare the red points to the green points, which correspond to $p<.10$ and $p<.005$. For smaller sample sizes, the red points achieve better performance than the green points, but for larger sample sizes, the relationship flips and the green points achieve better performance. This repeats the point made earlier that at larger sample sizes, a lower alpha should be used. For Bayes factors, compare the light blue and purple points, which correspond to Bayes factor thresholds of 1 and 3. For smaller sample sizes, the light blue points achieve better performance, but for larger sample sizes, the purple points achieve better performance. However, unlike with p-values, this reversal is not nearly as dramatic, and the threshold of Bayes factor $>1$ performs better than or nearly as good as the other thresholds across all sample sizes. It is also worth noting that as sample size increases, all Bayes factor criteria continue to improve, whereas pvalues plateau at their alpha levels. Thus, another advantage of Bayes factors is that increasing the amount of evidence increases their ability to accurately detect an effect. 


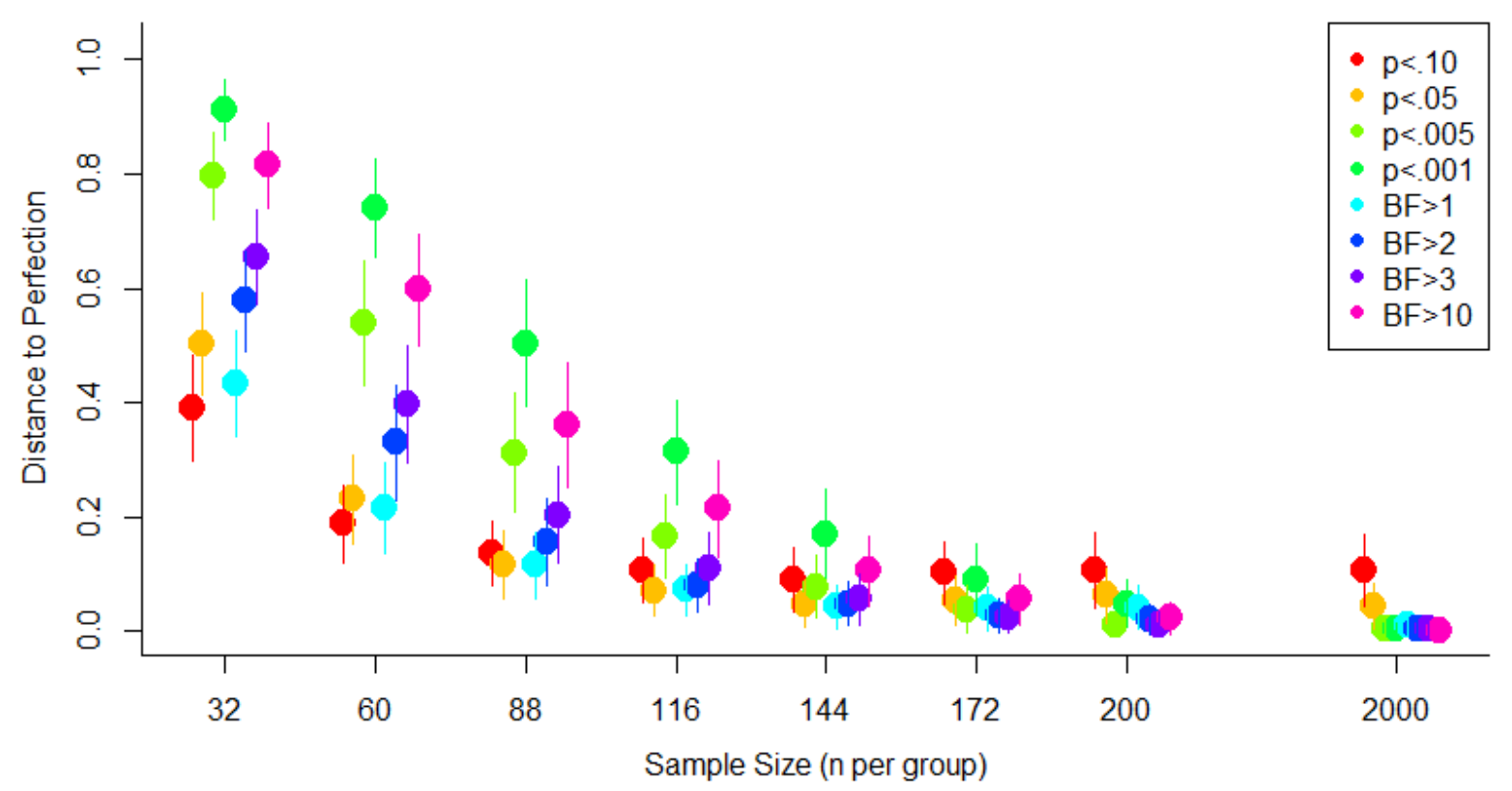

Figure 10. Distance to perfection was calculated as the Euclidean distance between each point on the ROC curve (see Figure 2) and the top-left corner (which corresponds to $100 \%$ hit rate and $0 \%$ false alarm rate). Distance to perfection scores were calculated for each of 100 sets of 20 studies (half of which were modeled as a null effect and half of which were modeled with Cohen's $d=.5$ ) for each sample size. The data are grouped by sample size, and color corresponds to the criterion for statistical significance. Errors bars correspond to $95 \%$ confidence intervals.

Signal detection analysis is a tool that scientists can use to evaluate relative trade-offs across various criteria of statistical significance. This is not to say that scientists should only use or always use criteria for statistical significance (as opposed to estimations of effect size, for example), but that when a criterion for statistical significance is adopted, consideration should be made for both false alarms and misses. If the goal is to maximize optimal utility, given equal weight to hits and correct rejections (or, equivalently, equal tolerance for false alarms and misses), distance to perfection can be used to assess various criteria. In the case of a medium effect size with 64 participants per group, the criteria of $p<$ $.10, p<.05$, and $B F>1$ led to better performance than the criteria of $p<.005, B F>3$, and $B F>10$. As sample size increased, the criteria of $p<.005$ and all tested Bayes factor thresholds led to better performance than $p<.10$. 


\section{Generalizing to Other Scenarios and Methodological Questions}

The performance measures of AUC and distance to perfection could be used to assess the best criteria for statistical significance for other situations, such as across different effects sizes, different underlying distributions, or different statistical tests such as one-sample t-tests, correlations, and ANOVAs (see Figures 11 and 12 for some examples). The technique could also be used to assess whether one study at $95 \%$ power provides better discrimination than one study and a replication at $80 \%$ power each (see Figure 13). If there is unequal tolerance with a desire to minimize false alarms at the expense of increasing misses, distance to perfection can be calculated by differentially weighting the costs of false alarms versus misses. The important point is that scientists (and journal editors) consider these trade-offs, rather than false alarm rates alone, when deciding which criterion to adopt.

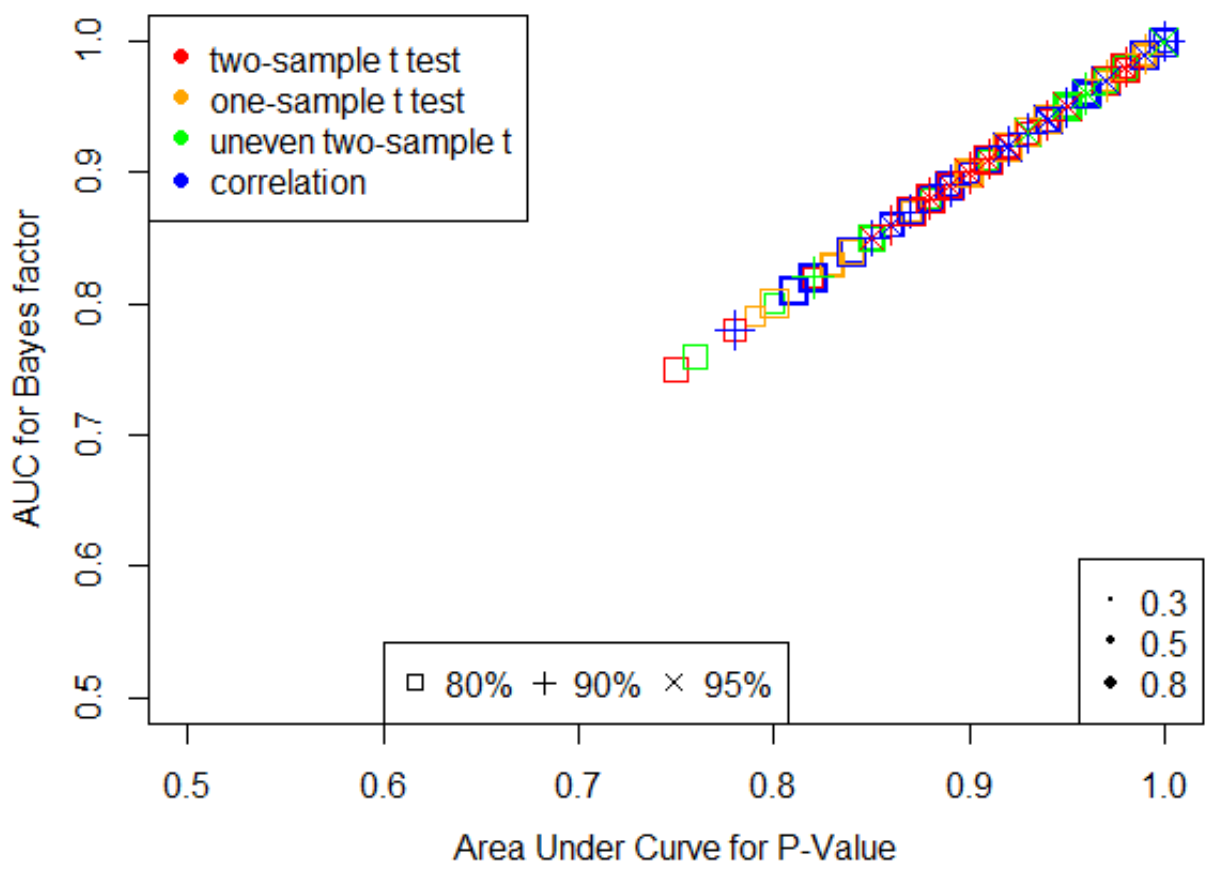

Figure 11. Simulations were run for 20 studies (repeated 100 times) for 3 effect sizes for 3 power levels (two-tailed at alpha $=.05$ ) for 4 types of statistical tests. AUCs for the Bayes factors are plotted as a function of AUCs for the p-values. They are identical in every case, which is consistent with the claims of equal discriminability between $p$-values and Bayes factors. Size of the symbol corresponds to effect size, which is Cohen's $d$ (for two-sample t-tests), Cohen's $d_{z}$ (for one-sample t-tests), and $r^{*} 2$ (for correlations). For the uneven two-sample t test, group 2 had $20 \%$ more participants than group 1. 

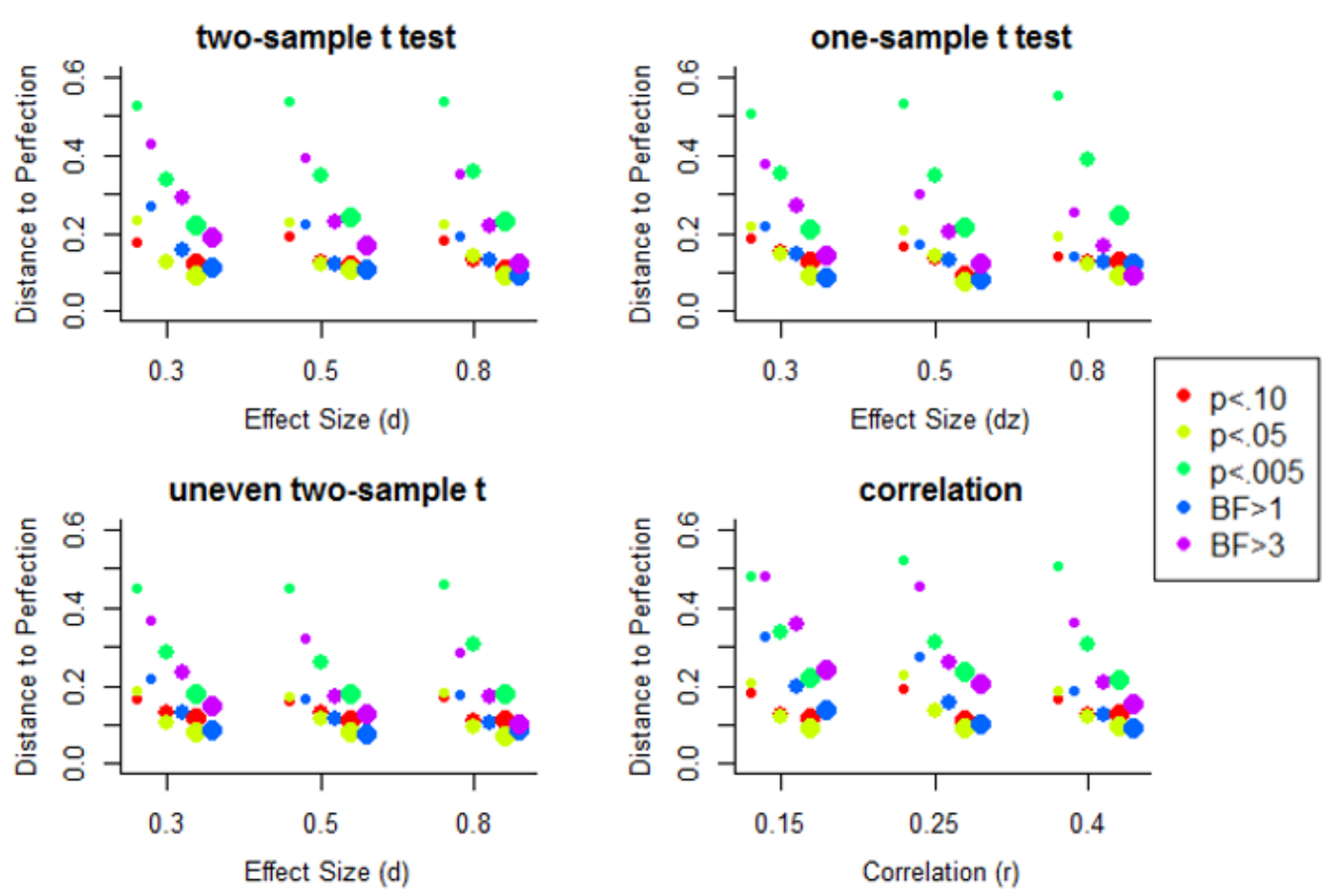

Figure 12. Simulations were run for 20 studies (repeated 100 times) for 3 effect sizes for 3 power levels (at alpha $=.05$ ) for 4 types of statistical tests. Distance to perfection was calculated as the Euclidean distance between each point on the ROC curve (see Figure 2) and the top-left corner (which corresponds to $100 \%$ hit rate and $0 \%$ false alarm rate). Sample size as a function of power $(80 \%, 90 \%, 95 \%)$ corresponds to the size of the points. Data points are clustered by modeled effect size (small differences in position along $\mathrm{x}$-axis are meaningless; data merely spread out to increase visibility).
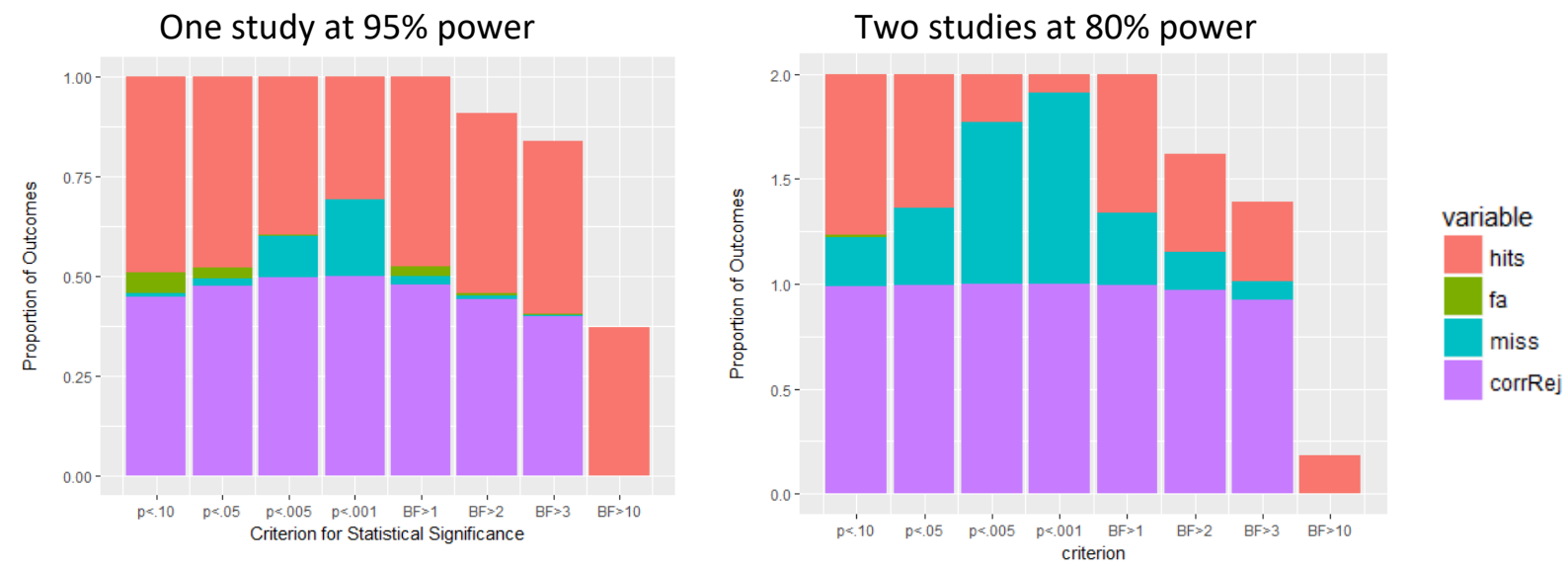

Figure 13. Proportion of each outcome as a function of the criterion for significance and whether one or two studies were run. The left panel shows the outcomes across 100 sets of 20 studies, each with 105 data points per group (which corresponds to $95 \%$ power at alpha $=.05$ ). The right panel shows the outcomes across 100 sets of 20 studies followed by 20 replications, each with 64 data points per group $(80 \%$ power at alpha $=.05)$. In order for an effect to be 
considered significant, both the original study and the replication had to produce values that exceeded the criterion for statistical significance. For example, for the criterion of $p<.05$, both the study and the replication had to produce $p$-values $<.05$, otherwise the set of studies was considered not significant.

\section{Discriminability with Effect Size}

As a final note, discriminability (as measured using AUCs) is as good or better when using effect size (in this case, Cohen's d) than p-values or Bayes factors (see Figure 14). This improved discriminability occurred because Cohen's $d$ is signed (i.e. differentiates -.5 from .5). When discriminability was assessed using absolute effect size, the AUCs matched those obtained with p-values and Bayes factors.

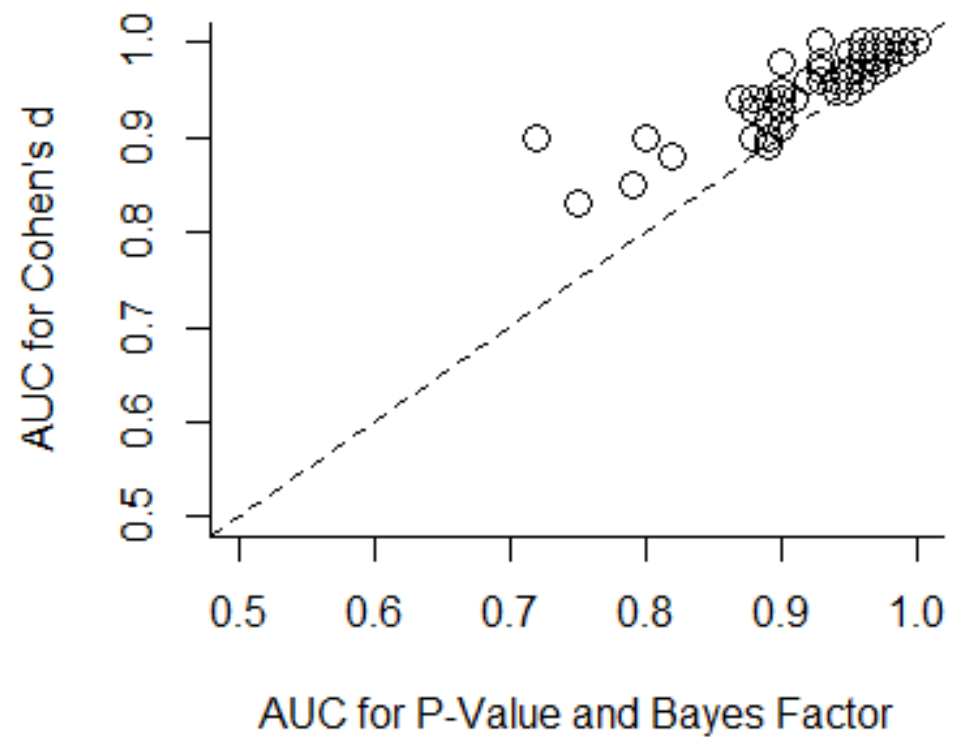

Figure 14. Area under the curve (AUC) for Cohen's $d$ as a function of the AUC for $p$-values and Bayes factors. Each point corresponds to one set of 20 studies with half modeled with Cohen's $d$ $=.5$ and half modeled with Cohen's $d=0$. Dotted line is at unity.

\section{Conclusion}

An essential part of science is that it is replicable. But another essential part of science is to uncover new discoveries. Changing the standard criterion for statistical significance merely moves the standard along the ROC curve. Any change to this standard such as decreasing the required $p$-value or 
using Bayes factors instead will not improve discriminability between real and null effects. Rather, a change to be more conservative will decrease false alarm rates at the expense of increasing miss rates. False alarm rates should not be considered in isolation without also considering miss rates. In addition, given that true null results can be theoretically interesting and practically important, a conservative criterion can produce critically misleading interpretations by labeling real effects as if they were null effects. Moving forward, the recommendation is to acknowledge the relationship between false alarms and misses, rather than implement standards based solely on false alarm rates. 


\section{Author Note}

Jessica K. Witt, Department of Psychology, Colorado State University, Fort Collins, CO 80523.

The author would like to thank Anne Cleary, John Wixted, Mark Prince, Susan Wagner Cook, Mike Dodd, Art Glenberg, Jim Nairne, Jeremy Wolfe, and Ben Prytherch for useful discussions and feedback on an earlier draft. This work was supported by grants from the National Science Foundation to JKW (BCS-1348916 and BCS-1632222).

Please address correspondence to Jessica Witt, Colorado State University, Behavioral Sciences Building, Fort Collins, CO 80523 USA. Email: Jessica.Witt@colostate.edu. 


\section{References}

Begley, C. G., \& Ellis, L. M. (2012). Drug development: Raise standards for preclinical cancer research. Nature, 483(29 March), 531-533.

Benjamin, D. J., Berger, J. O., Johannesson, M., Nosek, B. A., Wagenmakers, E. J., Berk, R., ... Johnson, V. E. (2017). Redefine statistical significance. Nature Human Behaviour. doi: 10.1038/s41562-017$0189-z$

Camerer, C. F., Dreber, A., Forsell, E., Ho, T.-H., Huber, J., Johannesson, M., . . Wu, H. (2016). Evaluating replicability of laboratory experiments in economics. Science, 351(6280), 1433-1436.

Collaboration, O. S. (2015). Estimating the reproducibility of psychological science. Science, 349(6251), aac4716.

Cumming, G. (2014). The new statistics: Why and how. Psychological Science, 25(1), 7-29.

Dienes, Z. (2011). Bayesian versus orthodox statistics: Which side are you on? Perspectives on Psychological Science, 6, 274-290.

Etz, A., \& Vandekerckhove, J. (2016). A Bayesian perspective on the reproducibility project: Psychology. PLos One, 11(2), e0149794.

Gilbert, D. T., King, G., Pettigrew, S., \& Wilson, T. D. (2016). Comment on "estimating the reproducibility of psychological science". Science, 351(6277), 1037-a.

Green, D. M., \& Swets, J. A. (1966). Signal Detection Theory and Psychophysics. New York: Wiley.

loannidis, J. P. A. (2005). Why most published research findings are false. PLoS Med, 2(8), e124.

Jefferys, W. H. (1990). Bayesian analysis of random event generator data. Journal of Scientific Exploration, 4(2), 153-169.

Jeffreys, H. (1961). Theory of Probability. Oxford, UK: Oxford University Press. 
Kass, R. E., \& Raftery, A. E. (1995). Bayes factors. Journal of the American Statistical Association, 90(430), 773-795.

Kruschke, J. K. (2011). Bayesian assessment of null values via parameter estimation and model comparison. Perspectives on Psychological Science, 6, 299-312.

Lindley, D. V. (1957). A statistical paradox. Biometrika, 44(1/2), 187-192.

Macmillan, N. A., \& Creelman, C. D. (2008). Detection Theory: A User's Guide (Second Edition). New York: Psychology Press.

Morey, R. D., Rouder, J. N., \& Jamil, T. (2014). BayesFactor: Computation of Bayes factors for common designs (Version 0.9.8). from http://CRAN.R-project.org/package=BayesFactor

Pisano, E. D., Gatsonis, C., Hendrick, E., Yaffe, M., Baum, J. K., Achayya, S., . . Rebner, M. (2005). Diagnostic performance of digital versus film mammography for breast-cancer screening. New England Journal of Medicine, 353(1773-1783).

Rouder, J. N., Speckman, P. L., Sun, D., Morey, R. D., \& Iverson, G. (2009). Bayesian t-tests for accepting and rejecting the null hypothesis. Psychonomic Bulletin \& Review, 16, 225-237.

Simmons, J. P., Nelson, L. D., \& Simonsohn, U. (2011). False-positive psychology: Undisclosed flexibility in data collection and analysis allows presenting anything as significant. Psychological Science, 22(11), 1359-1366.

Stern, H. S. (2016). A test by any other name: P-values, Bayes factors and statistical inference. Multivariate Behavioral Research, 51(1), 23-29.

Team, R. C. (2017). R: A language and environment for statistical computing. Retrieved from https://www.r-project.org

Trafimow, D., \& Marks, M. (2015). Editorial. Basic and Applied Social Psychology, 37(1), 1-2.

Van Bavel, J. J., Mende-Siedlecki, P., Brady, W. J., \& Reinero, D. A. (2016). Contextual sensitivity in scientific reproducibility. Proceedings of the National Academy of Sciences, 113(23), 6454-6459. 
Wetzels, R., Matzke, D., Lee, M. D., Rouder, J. N., Iverson, G. J., \& Wagenmakers, E.-J. (2011). Statistical evidence in experimental psychology: An empirical comparison using $855 \mathrm{t}$ tests. Perspectives on Psychological Science, 6(3), 291-298. 